\title{
APPLICATIONS OF THE LOCAL ALGEBRAS OF VECTOR FIELDS TO THE MODELLING OF PHYSICAL PHENOMENA
}

\author{
IGOR V. BAYAK
}

Communicated by Izu Vaisman

\begin{abstract}
In this paper we discuss the local algebras of linear vector fields that can be used in the mathematical modelling of physical space by building the dynamical flows of vector fields on eight-dimensional cylindrical or toroidal manifolds. It is shown that the topological features of the vector fields obey the Dirac equation when moving freely within the surface of a pseudo-sphere in the eight-dimensional pseudo-Euclidean space.
\end{abstract}

MSC: 57R25

Keywords: vector fields, algebra of linear vector fields, dynamic flow, topological features

\section{Contents}

1 Introduction 1

2 Local Algebra of Vector Fields 4

3 The Geometry of the Algebra of Vector Fields 9

4 The Dynamics of the Algebra of Vector Fields 14

References

\section{Introduction}

The paper contains a collection of algebraic, geometric and dynamical facts concerning linear vector fields on simple locally affine manifolds ( $\mathbb{R}^{n}$, cylinders, tori) where the main ingredient is the algebra defined by these vector fields with the product $X \star Y=\nabla_{X} Y$, where $\nabla$ is the flat, torsionless connection of the locally affine structure of the manifold. 
Definition 1. Let $X$ be a vector field on a smooth manifold $M$, where $(U, u)$ is a local chart of $M$ and $\left\{u^{i}\right\}$ are the local coordinates system associated with it. A vector field $X$ with its local representation

$$
X=X^{i} \frac{\partial}{\partial u^{i}}
$$

is called linear relative to $\left\{u^{i}\right\}$ if its local components are of the form

$$
X^{i}=C_{j}^{i} u^{j}
$$

where $C_{j}^{i} \in \mathbb{R}$.

Starting with the simplest two-dimensional case, we recall that if smooth vector fields

$$
A(x, y)=a_{x}(x, y) \partial_{x}+a_{y}(x, y) \partial_{y}, \quad B(x, y)=b_{x}(x, y) \partial_{x}+b_{y}(x, y) \partial_{y}
$$

together with a function $f(x, y)$ are defined on the Cartesian plane $(x, y)$ then the gradient of the function $f(x, y)$ is a differential one-form (a covector field)

$$
\nabla f(x, y)=\frac{\partial f(x, y)}{\partial x} \mathrm{~d} x+\frac{\partial f(x, y)}{\partial y} \mathrm{~d} y
$$

a derivative of $f(x, y)$ in the direction of the vector field $A(x, y)$ is a function of the convolution of the gradient $\nabla f(x, y)$ and the vector field $A(x, y)$

$$
\nabla_{A} f(x, y)=\langle A(x, y), \nabla f(x, y)\rangle=a_{x} \frac{\partial f(x, y)}{\partial x}+a_{y} \frac{\partial f(x, y)}{\partial y} .
$$

In turn, a derivative of the vector field $B(x, y)$ along a vector field $A(x, y)$ is a vector field

$$
\nabla_{A} B=\nabla_{A} b_{x} \partial_{x}+\nabla_{A} b_{y} \partial_{y} .
$$

Respectively, a derivative of the field $A(x, y)$ in the direction of $B(x, y)$ is a vector field

$$
\nabla_{B} A=\nabla_{B} a_{x} \partial_{x}+\nabla_{B} a_{y} \partial_{y} .
$$

Finally, the Lie bracket of two vector fields is a vector field

$$
[A, B]=\nabla_{A} B-\nabla_{B} A=\left(\nabla_{A} b_{x}-\nabla_{B} a_{x}\right) \partial_{x}+\left(\nabla_{A} b_{y}-\nabla_{B} a_{y}\right) \partial_{y} .
$$

Let all the vector fields considered hereafter be linear [7], i.e., their coordinate functions are linear functions of the coordinates of the Cartesian plane. Then the linear vector fields of the Cartesian plane, with the derivative of a vector field in the direction of another vector field as product, form the algebra $\mathcal{A}_{\nabla}$, which is 
isomorphic to the matrix algebra $M_{2}(\mathbb{R})$ and is invariant under non-degenerate linear transformations of the Cartesian plane coordinates. However, the linear tangent vector fields (i.e., linear vector fields without a radial component, which are collinear unit vector field $x \partial_{x}+y \partial_{y}$ ) with respect to the Lie bracket form an algebra $\mathcal{A}_{T}$, which is isomorphic to the matrix algebra $\operatorname{Lie}_{\mathfrak{s}}(\mathbb{R})$ and is invariant with respect to arbitrary non-degenerate linear transformations of the coordinates of the Cartesian plane.

As an illustration of these algebras we construct an algebra of linear vector fields with the structure of the algebra of complex numbers. Take the vector fields

$$
E=x \partial_{x}+y \partial_{y}, \quad I=y \partial_{x}-x \partial_{y}
$$

for which the following relations hold

$$
\begin{array}{r}
E^{2}=E \star E=\nabla_{E} E=x \partial_{x}+y \partial_{y}=E \\
E \star I=I \star E=\nabla_{E} I=\nabla_{I} E=y \partial_{x}-x \partial_{y}=I \\
I^{2}=I \star I=\nabla_{I} I=-x \partial_{x}-y \partial_{y}=-E .
\end{array}
$$

Hence the vector field $I$ is the generator of the algebra of linear vector fields (subalgebras $\left.\mathcal{A}_{\nabla}\right)$, which is isomorphic to the algebra of complex numbers: $\langle(I, \star)\rangle_{\mathbb{R}} \cong$ $\mathbb{C}$, and the Lie algebra of linear vector fields (subalgebras $\mathcal{A}_{T}$ ), which is isomorphic to the corresponding unitary algebra $\langle(I,[,])\rangle_{\mathbb{R}} \cong \mathfrak{u}(1)$.

The notion of an algebra $\mathcal{A}_{\nabla}$ of linear vector fields on the Cartesian plane can be easily generalized to the case of the Cartesian space $\mathbb{R}^{n}$. Indeed, if we take an arbitrary linear vector field

$$
A=a^{1} \partial x_{1}+\cdots+a^{n} \partial x_{n}=a^{i} \partial x_{i}
$$

where $a^{i}=a_{i 1} x^{1}+\cdots+a_{i n} x^{n}$, and an arbitrary linear vector field

$$
B=b^{1} \partial x_{1}+\cdots+b^{n} \partial x_{n}=b^{i} \partial x_{i}
$$


where $b^{i}=b_{i 1} x^{1}+\cdots+b_{i n} x^{n}$, and define the operation of differentiation of a vector field $A$ in the direction of the vector field $B$

$$
\begin{aligned}
\nabla_{B} A= & \sum_{i=1}^{n} \nabla_{B} a^{i} \partial x_{i}=\sum_{i=1}^{n} b^{j} \frac{\partial a^{i}}{\partial x_{j}} \partial x_{i} \\
= & \sum_{i=1}^{n}\left(b_{11} x^{1}+\cdots+b_{1 n} x^{n}\right) \frac{\partial\left(a_{i 1} x^{1}+\cdots+a_{i n} x^{n}\right)}{\partial x_{1}} \partial x_{i} \\
& +\cdots+\left(b_{n 1} x^{1}+\cdots+b_{n n} x^{n}\right) \frac{\partial\left(a_{i 1} x^{1}+\cdots+a_{i n} x^{n}\right)}{\partial x_{n}} \partial x_{i} \\
= & \sum_{i=1}^{n}\left[\left(a_{i 1} b_{11}+\cdots+a_{i n} b_{n 1}\right) x^{1}+\cdots+\left(a_{i 1} b_{1 n}+\cdots+a_{i n} b_{n n}\right) x^{n}\right] \partial x_{i} \\
= & \sum_{i=1}^{n} c_{i j} x^{j} \partial x_{i}=c^{i} \partial x_{i}=C
\end{aligned}
$$

then, since $C=\left(c_{i j}\right)_{n}=\left(a_{i j}\right)_{n}\left(b_{i j}\right)_{n}=A B \cong \nabla_{B} A=B \star A$, we find an equivalence between the algebra of linear vector fields in Cartesian space $\mathbb{R}^{n}$ and the matrix algebra $M_{n}(\mathbb{R})$, which is the algebra of endomorphisms of a linear space $\mathbb{R}^{n}$. Note also that we consider below only subalgebras of linear vector fields.

\section{Local Algebra of Vector Fields}

Let an element of the local algebra of linear vector fields $\mathcal{A}_{\nabla}\left(T_{x^{\prime}} M^{n}\right)$ be defined on the tangent bundle of the manifold $M^{n}$ through the following formal expression

$$
c\left(x^{\prime}\right)=\sum_{i=1}^{n} c_{i j}\left(x^{\prime}\right) \Delta x^{\prime j} \partial x_{i}^{\prime}
$$

where $\left(c_{i j}\right)_{n}=C \in M_{n}(\mathbb{R})$, and $M_{n}(\mathbb{R})$ - is the matrix algebra of endomorphisms of $\mathbb{R}^{n}, \partial x_{i}^{\prime} \in T_{x^{\prime}} M^{n}$, and $\Delta x^{\prime j}$ are the coordinates of an arbitrary point in the tangent space $T_{x^{\prime}} M^{n}$. Then, an element of the local algebra of linear vector fields on the tangent bundle of the space $\mathbb{R}^{n}$ is induced by the non-degenerate map $F: \mathbb{R}^{n} \mapsto M^{n}:\left(x \rightarrow x^{\prime}\right)$ from $\mathcal{A}_{\nabla}\left(T_{x^{\prime}} M^{n}\right)$ to $\mathcal{A}_{\nabla}\left(\mathbb{R}^{n}\right)$. Indeed, since $\partial x_{i} \in T_{x} \mathbb{R}^{n}, \Delta x^{\prime j}=J_{j i}(x) \Delta x^{i}, \partial x_{i}^{\prime}=J_{i j}^{-1}(x) \partial x_{j}$, where $J_{i j}(x)$ is the Jacobian of the map $F$, then

$$
c^{\prime}(x)=\sum_{i=1}^{n}\left(J^{-1} C J\right)_{i j}(x) \Delta x^{j} \partial x_{i}
$$


with $c^{\prime}(x) \in \mathcal{A}_{\nabla}\left(T_{x^{\prime}} \mathbb{R}^{n}\right)$. Conversely, if we already have a local algebra $\mathcal{A}_{\nabla}\left(\mathbb{R}^{n}\right)$ such that

$$
c^{\prime}(x)=\sum_{i=1}^{n} c_{i j}^{\prime}(x) \Delta x^{j} \partial x_{i}
$$

then, in virtue of $C=J C^{\prime} J^{-1}$, this algebra is transferred to $\mathcal{A}_{\nabla}\left(T_{x^{\prime}} M^{n}\right)$ in the form of the following expression

$$
c\left(x^{\prime}\right)=\sum_{i=1}^{n}\left(J C^{\prime} J^{-1}\right)_{i j}\left(x^{\prime}\right) \Delta x^{\prime j} \partial x_{i}^{\prime} .
$$

Besides, in the case of the algebra $\mathcal{A}$ being a subalgebra of the matrix algebra of endomorphisms of $\mathbb{R}^{n}$ and for all $x^{\prime}$ being fulfilled $C\left(x^{\prime}\right) \in \mathcal{A}$, some additional conditions should be imposed on the elements of the local algebra $\mathcal{A}_{\nabla}\left(T_{x^{\prime}} M^{n}\right)$. Indeed, if $C\left(x^{\prime}\right), C^{\prime}(x) \in \mathcal{A}$ for all $x^{\prime}, x$, then $J \in G \mathcal{A}$ where $G \mathcal{A}$ is the group of invertible elements of the subalgebra $\mathcal{A}$, since only $J$ acts on the algebra $\mathcal{A}$, according to the formula of inner automorphisms of the group $G \mathcal{A}$ : $C^{\prime}=J^{-1} C J$. In this case we deal with a global algebra $\mathcal{A}_{\nabla}\left(M^{n}\right)$ on the locally affine manifold $M^{n}$.

For example, when the condition $J \in G \mathcal{A}$ is satisfied, and if we take a subalgebra of linear vector fields $\mathcal{A}_{\nabla}\left(\mathbb{R}^{2}\right)$ with the structure of the algebra of complex numbers, then we obtain the Cauchy-Riemann conditions. In fact, if $C^{\prime}(x) \in \mathbb{C}$, then the following condition

$$
J(x)=\left(\begin{array}{ll}
\frac{\partial x_{1}^{\prime}}{\partial x_{1}} & \frac{\partial x_{1}^{\prime}}{\partial x_{2}} \\
\frac{\partial x_{2}^{\prime}}{\partial x_{1}} & \frac{\partial x_{2}^{\prime}}{\partial x_{2}}
\end{array}\right) \in \mathbb{C} \backslash\{0\}
$$

has to be satisfied, which means that

$$
\frac{\partial x_{1}^{\prime}}{\partial x_{1}}=\frac{\partial x_{2}^{\prime}}{\partial x_{2}}, \quad \frac{\partial x_{1}^{\prime}}{\partial x_{2}}=-\frac{\partial x_{2}^{\prime}}{\partial x_{1}} .
$$

Similarly, if we take a subalgebra of $\mathcal{A}_{\nabla}\left(\mathbb{R}^{8}\right)$ with the structure of the matrix algebra $M_{4}(\mathbb{C})$, then by virtue of

$$
J(x)=\left(\begin{array}{ll}
\frac{\partial x_{2 i-1}^{\prime}}{\partial x_{2 j-1}} & \frac{\partial x_{2 i-1}^{\prime}}{\partial x_{2 j}} \\
\frac{\partial x_{2 i}^{\prime}}{\partial x_{2 j-1}} & \frac{\partial x_{2 i}^{\prime}}{\partial x_{2 j}}
\end{array}\right)_{8} \in M_{4}(\mathbb{C}) \backslash\{0\}
$$

where $i, j=1, \ldots, 4$, we obtain the Cauchy-Riemann conditions

$$
\frac{\partial x_{2 i-1}^{\prime}}{\partial x_{2 j-1}}=\frac{\partial x_{2 i}^{\prime}}{\partial x_{2 j}}, \quad \frac{\partial x_{2 i-1}^{\prime}}{\partial x_{2 j}}=-\frac{\partial x_{2 i}^{\prime}}{\partial x_{2 j-1}} .
$$


Suppose now we have

$$
\begin{gathered}
x_{1}=\mathrm{e}^{z} \cos \varphi, \quad x_{2}=\mathrm{e}^{z} \sin \varphi \\
C^{\prime}(x)=\left(\begin{array}{rr}
x_{1} & -x_{2} \\
x_{2} & x_{1}
\end{array}\right) \in \mathbb{C} .
\end{gathered}
$$

Then the map $F: \mathbb{R}^{2} \mapsto \mathbb{R} \times S^{1}:\left(x_{1}, x_{2}\right) \rightarrow(z, \varphi)$ has the Jacobian

$$
J(z, \varphi)=\mathrm{e}^{-z}\left(\begin{array}{rr}
\cos \varphi & \sin \varphi \\
-\sin \varphi & \cos \varphi
\end{array}\right) \in \mathbb{C} \backslash\{0\}
$$

and, therefore

$$
C(z, \varphi)=J C^{\prime}(x) J^{-1}=\mathrm{e}^{z}\left(\begin{array}{rr}
\cos \varphi & \sin \varphi \\
-\sin \varphi & \cos \varphi
\end{array}\right) \in \mathbb{C} .
$$

Thus, a complex plane regarded as a subalgebra of $\mathcal{A}_{\nabla}\left(\mathbb{R}^{2}\right)$ with the structure of $\mathbb{C}$ and a complex cylinder regarded as a subalgebra of $\mathcal{A}_{\nabla}\left(\mathbb{R} \times S^{1}\right)$ with the structure of $\mathbb{C}$ are equivalent to each other, with the correspondence between them being equivalent to a complex-analytical map

$$
z+\mathrm{i} \varphi \rightarrow \mathrm{e}^{z} \mathrm{e}^{\mathrm{i} \varphi}:\left(\begin{array}{cc}
z & -\varphi \\
\varphi & z
\end{array}\right) \rightarrow \mathrm{e}^{z}\left(\begin{array}{rr}
\cos \varphi & \sin \varphi \\
-\sin \varphi & \cos \varphi
\end{array}\right)
$$

where $z, \varphi \in \mathbb{R}$, in which the complexification of the covering of the cylinder is mapped onto the complexification of the cylinder itself.

Now let a subalgebra of linear vector fields $\mathcal{A}_{\nabla}\left(M^{\prime 8}\right)$ be induced by the map $F$ : $\left.M^{8} \mapsto M^{\prime 8}:\left(z_{i}, \varphi_{i}\right)_{4} \rightarrow\left(z_{i}^{\prime}, \varphi_{i}^{\prime}\right)_{4}\right)$ with the Jacobian $J\left(z^{\prime}, \varphi^{\prime}\right)$. Then the CauchyRiemann equations would be

$$
\frac{\partial z_{i}^{\prime}}{\partial z_{j}}=\frac{\partial \varphi_{i}^{\prime}}{\partial \varphi_{j}}, \quad \frac{\partial z_{i}^{\prime}}{\partial \varphi_{j}}=-\frac{\partial \varphi_{i}^{\prime}}{\partial z_{j}}
$$

where $i, j=1,2,3,4$.

We can also note that, if $C^{\prime}(x)$ belongs to the algebra of dual numbers

$$
C^{\prime}(x)=\left(\begin{array}{ll}
x_{1} & x_{2} \\
x_{2} & x_{1}
\end{array}\right) \in \mathbb{D}
$$

then, in order to satisfy the belonging condition $C\left(x^{\prime}\right) \in \mathbb{D}$, it is required that the following hyperbolic Cauchy-Riemann equations hold

$$
\frac{\partial x_{1}^{\prime}}{\partial x_{1}}=\frac{\partial x_{2}^{\prime}}{\partial x_{2}}, \quad \frac{\partial x_{1}^{\prime}}{\partial x_{2}}=\frac{\partial x_{2}^{\prime}}{\partial x_{1}}
$$


Let us now consider an application of the local algebra of linear vector fields to the case when this algebra is used to represent the Riemann zeta function. First of all, we should note that the flow of the linear vector field on the Cartesian plane which is represented by the matrix

$$
C=\left(\begin{array}{rr}
a & -b \\
b & a
\end{array}\right)
$$

has a spiral shape described by the following parametric equation:

$$
x_{1}(t)=\mathrm{e}^{a t+z_{0}} \cos \left(b t+\varphi_{0}\right), \quad x_{2}(t)=\mathrm{e}^{a t+z_{0}} \sin \left(b t+\varphi_{0}\right)
$$

with $x_{1}(0)=\mathrm{e}^{z_{0}} \cos \varphi_{0}$ and $x_{2}(0)=\mathrm{e}^{z_{0}} \sin \varphi_{0}$. On a cylinder, they have a helical shape described as

$$
z(t)=a t+z_{0}, \quad \varphi(t)=\left|b t+\varphi_{0}\right|_{2 \pi}
$$

with $\dot{z}(t)=a, \dot{\varphi}(t)=b$. However, the mapping of a plane onto a torus $F: \mathbb{R}^{2} \mapsto$ $S^{1} \times S^{1}$ induces a complex analytic map

$$
z+\mathrm{i} \varphi \rightarrow\left|\mathrm{e}^{z}\right|_{2} \mathrm{e}^{\mathrm{i} \varphi}=|\rho|_{2} \mathrm{e}^{\mathrm{i} \varphi}
$$

where $z, \varphi \in \mathbb{R}, \rho \in \mathbb{R}^{+},|\rho|_{2} \in\left[0,2\left[,|\rho|_{2}: \rho^{\prime} \equiv \rho(\bmod 2)\right.\right.$, or a complex analytical map

$$
z+\mathrm{i} \varphi \rightarrow\left|\mathrm{e}^{z}\right|_{ \pm 1} \mathrm{e}^{\mathrm{i} \varphi}=\tilde{\rho} \mathrm{e}^{\mathrm{i} \varphi}
$$

where

$$
\tilde{\rho}=\left|\mathrm{e}^{z}\right|_{ \pm 1}=\left\{\begin{array}{l}
\left|\mathrm{e}^{z}\right|_{2} \text { if }\left|\mathrm{e}^{z}\right|_{2} \in[0,1] \\
\left.\left|\mathrm{e}^{z}\right|_{2}-2 \text { if }\left|\mathrm{e}^{z}\right|_{2} \in\right] 1,2[.
\end{array}\right.
$$

The above sawtooth-shaped function can be represented by a Fourier series

$$
|x|_{ \pm 1}=\frac{2}{\pi} \sum_{1}^{\infty} \frac{(-1)^{k+1}}{k} \sin (k x)
$$

In this case, a helical trajectory on the cylinder

$$
z(t)=a t, \quad \varphi(t)=|b t|_{2 \pi}
$$

is transformed into a spiral on the torus

$$
\tilde{\rho}(t)=\left|\mathrm{e}^{a t}\right|_{ \pm 1}, \quad \varphi(t)=|b t|_{2 \pi}
$$

whereas the sum

$$
S(a, b)=\sum_{1}^{\infty} \frac{1}{n}\left|\mathrm{e}^{t_{n}-a t_{n}}\right|_{ \pm 1} \mathrm{e}^{\mathrm{i} b t_{n}}=\sum_{n=1}^{\infty} \frac{2}{\pi n} \sum_{k=1}^{\infty} \frac{(-1)^{k+1}}{k} \sin \left(k n^{1-a}\right) n^{\mathrm{i} b}
$$


with $t_{n}=\ln (n)$ represents the Riemann zeta function $\zeta(s)$. Indeed, since

$$
S(s>1)=\sum_{1}^{\infty} \frac{1}{n^{s}}
$$

where $s=a-\mathrm{i} b$, then we only have to prove the convergence of the sum $S(s<1)$. This sum is convergent because of the fact that $|S(s<1)|$ is smaller than the sum of a harmonic series because

$$
\left.\left|\frac{1}{n}\right| \mathrm{e}^{(1-s) t_{n}}\right|_{ \pm 1} \mid<\frac{1}{n}
$$

is satisfied almost always.

Another example of using the local algebra of linear vector fields is the mathematical derivation of the equation of the vibrations of a cylindrical string.

Proposition 1. Let curvilinear coordinates on an infinite cylinder satisfy the CauchyRiemann equations

$$
\frac{\partial z^{\prime}}{\partial z}=\frac{\partial \varphi^{\prime}}{\partial \varphi}, \quad \frac{\partial z^{\prime}}{\partial \varphi}=-\frac{\partial \varphi^{\prime}}{\partial z}
$$

and let a function $\psi(x, t)=z^{\prime}(x, t)+\varphi^{\prime}(x, t)$ be defined with

$$
x=\mathrm{e}^{-\varepsilon} z-\mathrm{e}^{\varepsilon} \varphi, \quad t=\mathrm{e}^{-\varepsilon} z+\mathrm{e}^{\varepsilon} \varphi
$$

in such a way that $z^{\prime}(x, t) \cong 1$ and $\varphi^{\prime}(x, t) \cong \mathrm{i}$. That is, the function $\psi(x, t)$ and its partial derivatives are equivalent to an element $1+\mathrm{i}$ of the algebra of complex numbers. Then the function $\psi(x, t)=z^{\prime}(x, t)+\varphi^{\prime}(x, t)$ satisfies the equation

$$
\mathrm{e}^{\mathrm{i} \alpha} \frac{\partial^{2} \psi}{\partial t^{2}}+\mathrm{e}^{\mathrm{i}(\alpha+\pi)} \frac{\partial^{2} \psi}{\partial x^{2}}=0
$$

where $\alpha=\sinh 2 \varepsilon$.

Proof: Indeed, as the following identities hold

$$
\begin{aligned}
\frac{\partial z^{\prime}}{\partial z} & =\mathrm{e}^{-\varepsilon} \frac{\partial z^{\prime}}{\partial x}+\mathrm{e}^{-\varepsilon} \frac{\partial z^{\prime}}{\partial t} \\
\frac{\partial z^{\prime}}{\partial \varphi} & =\mathrm{e}^{\varepsilon} \frac{\partial z^{\prime}}{\partial t}-\mathrm{e}^{\varepsilon} \frac{\partial z^{\prime}}{\partial x} \\
\frac{\partial \varphi^{\prime}}{\partial z} & =\mathrm{e}^{-\varepsilon} \frac{\partial \varphi^{\prime}}{\partial x}+\mathrm{e}^{-\varepsilon} \frac{\partial \varphi^{\prime}}{\partial t} \\
\frac{\partial \varphi^{\prime}}{\partial \varphi} & =\mathrm{e}^{\varepsilon} \frac{\partial \varphi^{\prime}}{\partial t}-\mathrm{e}^{\varepsilon} \frac{\partial \varphi^{\prime}}{\partial x}
\end{aligned}
$$


then the following conditions

$$
\begin{gathered}
\mathrm{e}^{\varepsilon} \frac{\partial z^{\prime}}{\partial x}-\mathrm{e}^{-\varepsilon} \frac{\partial \varphi^{\prime}}{\partial x}=\mathrm{e}^{\varepsilon} \frac{\partial z^{\prime}}{\partial t}+\mathrm{e}^{-\varepsilon} \frac{\partial \varphi^{\prime}}{\partial t} \\
-\mathrm{e}^{-\varepsilon \frac{\partial z^{\prime}}{\partial t}+\mathrm{e}^{\varepsilon} \frac{\partial \varphi^{\prime}}{\partial t}}=\mathrm{e}^{-\varepsilon} \frac{\partial z^{\prime}}{\partial x}+\mathrm{e}^{\varepsilon} \frac{\partial \varphi^{\prime}}{\partial x}
\end{gathered}
$$

are satisfied. Then, after differentiating the first equation with respect to $t$, and the second - with respect to $x$, we obtain a system of differential equations

$$
\begin{gathered}
\mathrm{e}^{\varepsilon} \frac{\partial^{2} z^{\prime}}{\partial x \partial t}-\mathrm{e}^{-\varepsilon} \frac{\partial^{2} \varphi^{\prime}}{\partial x \partial t}=\mathrm{e}^{\varepsilon} \frac{\partial^{2} z^{\prime}}{\partial t^{2}}+\mathrm{e}^{-\varepsilon} \frac{\partial^{2} \varphi^{\prime}}{\partial t^{2}} \\
-\mathrm{e}^{-\varepsilon} \frac{\partial^{2} z^{\prime}}{\partial t \partial x}+\mathrm{e}^{\varepsilon} \frac{\partial^{2} \varphi^{\prime}}{\partial t \partial x}=\mathrm{e}^{-\varepsilon} \frac{\partial^{2} z^{\prime}}{\partial x^{2}}+\mathrm{e}^{\varepsilon} \frac{\partial^{2} \varphi^{\prime}}{\partial x^{2}}
\end{gathered}
$$

which is equivalent to the system

$$
\bar{c} \frac{\partial^{2} \psi}{\partial x \partial t}=c \frac{\partial^{2} \psi}{\partial t^{2}}, \quad-\bar{b} \frac{\partial^{2} \psi}{\partial t \partial x}=b \frac{\partial^{2} \psi}{\partial x^{2}}
$$

where $c=\cosh \varepsilon+\mathrm{i} \sinh \varepsilon, b=\sinh \varepsilon+\mathrm{i} \cosh \varepsilon$. By multiplying the first equation of the system (48) by $c$, the second equation by $b$, and then by adding the resulting equations to each other we obtain the equation of oscillations of a cylindrical string (44) if we take into account the fact that $c^{2}=\mathrm{e}^{\mathrm{i} \sinh 2 \varepsilon}, b^{2}=\mathrm{e}^{\mathrm{i}(\sinh 2 \varepsilon+\pi)}$.

Corollary 1. In the case of $\sinh 2 \varepsilon=2 \pi k$, where $k \in \mathbb{Z}$, the equation of an oscillating cylindrical string (44) has the form

$$
\frac{\partial^{2} \psi}{\partial t^{2}}-\frac{\partial^{2} \psi}{\partial x^{2}}=0
$$

\section{The Geometry of the Algebra of Vector Fields}

First of all, let us turn to the question of the representation of multidimensional spaces that preserve quadratic forms. If only one quadratic form is preserved in a n-dimensional linear space, this form takes the canonical form

$$
x_{1}^{2}+\cdots+x_{k}^{2}-x_{k+1}^{2}-\cdots-x_{m}^{2}
$$

where $n-m=d, m-k=l$. Then, following the approach of [6], we can represent this space as a semi-Euclidean space with defect $d$ and index $l$, which is denoted as $l+\{d\} \mathbb{R}_{n}$. In the zero-defect case we are dealing with the Euclidean spaces of index $l$. However, if we impose the condition of preserving several quadratic forms in 
the $n$-dimensional linear space, the representation becomes ambiguous. Firstly, we have to distinguish the condition of simultaneous preservation of quadratic forms and the condition of the preservation of these forms separately from each other. For example, if, in a two-dimensional linear space, either the form $x_{1}^{2}+x_{2}^{2}$ or the form $x_{1}^{2}-x_{2}^{2}$ is preserved, then we consider our space as a system of two Euclidean spaces $\left\{{ }^{1} \mathbb{R}_{2}, \mathbb{R}_{2}\right\}$. However, if a system of equations

$$
x_{1}^{2}+x_{2}^{2}=\text { const }, \quad x_{3}^{2}+x_{4}^{2}=\text { const }
$$

has to be satisfied in the four-dimensional linear space then we consider this space as a joint system of two semi-Euclidean spaces $\left\{{ }^{\{2\}} \mathbb{R}_{4},{ }^{\{2\}} \mathbb{R}_{4}\right\}$. Secondly, it should be realized that a joint system of equations corresponds to the whole class of equivalence. For example, the system (51) is equivalent to the system

$$
x_{1}^{2}+x_{2}^{2}+x_{3}^{2}+x_{4}^{2}=\text { const }, \quad x_{1}^{2}+x_{2}^{2}-x_{3}^{2}-x_{4}^{2}=\text { const }
$$

which corresponds to the representation of a joint system of Euclidean spaces $\left\{{ }^{2} \mathbb{R}_{4}, \mathbb{R}_{4}\right\}$ and, hence, the representations $\left\{2^{\{2\}} \mathbb{R}_{4}\right\} \cong\left\{{ }^{2} \mathbb{R}_{4}, \mathbb{R}_{4}\right\}$ are equivalent. Similarly, due to the equivalence of the system

$$
\begin{aligned}
x_{1}^{2}+x_{2}^{2}=\text { const }, & x_{3}^{2}+x_{4}^{2}=\text { const } \\
x_{5}^{2}+x_{6}^{2}=\text { const }, & x_{7}^{2}+x_{8}^{2}=\text { const }
\end{aligned}
$$

and the system

$$
\begin{aligned}
& x_{1}^{2}+x_{2}^{2}+x_{3}^{2}+x_{4}^{2}+x_{5}^{2}+x_{6}^{2}+x_{7}^{2}+x_{8}^{2}=\mathrm{const} \\
& x_{1}^{2}+x_{2}^{2}-x_{3}^{2}-x_{4}^{2}+x_{5}^{2}+x_{6}^{2}-x_{7}^{2}-x_{8}^{2}=\mathrm{const} \\
& x_{1}^{2}+x_{2}^{2}+x_{3}^{2}+x_{4}^{2}-x_{5}^{2}-x_{6}^{2}-x_{7}^{2}-x_{8}^{2}=\mathrm{const} \\
& x_{1}^{2}+x_{2}^{2}-x_{3}^{2}-x_{4}^{2}-x_{5}^{2}-x_{6}^{2}+x_{7}^{2}+x_{8}^{2}=\mathrm{const}
\end{aligned}
$$

the representations $\left\{4^{\{6\}} \mathbb{R}_{8}\right\} \cong\left\{\mathbb{R}_{8}, 3^{4} \mathbb{R}_{8}\right\}$ are equivalent too.

Let us consider an algebra $\mathcal{A}_{\nabla}\left(\mathbb{R}_{2}\right)$ of linear vector fields with the structure of the algebra of complex numbers $\mathbb{C}$. The geometric structure of this algebra is defined by its generating vector fields, namely, by the radial and tangent vector fields

$$
E=x \partial_{x}+y \partial_{y}, \quad I=y \partial_{x}-x \partial_{y} .
$$

Since the vector field $I$ is orthogonal to $E$ in the metric of the Euclidean plane $(x, y)$ and, therefore, it is tangent to the circles $x^{2}+y^{2}=$ const, then in this case $\mathcal{A}_{\nabla}\left(\mathbb{R}_{2}\right)$ - is the algebra of linear vector fields of the Euclidean plane $\mathbb{R}_{2}$. 
Similarly, the geometric structure of algebra $\mathcal{A}_{\nabla}\left({ }^{1} \mathbb{R}_{2}\right)$ of the linear vector fields with the structure of the algebra of dual numbers $\mathbb{D}$ is determined by its generating vector fields, namely, by the radial and tangent vector fields

$$
E=x \partial_{x}+y \partial_{y}, \quad J=y \partial_{x}+x \partial_{y}
$$

where the vector field $J$ is orthogonal to $E$ in the metric of the pseudo-Euclidean plane $(x, y)$, and, hence, it is tangent to an equilateral hyperbola $x^{2}-y^{2}=$ const. Thus, in this case $\mathcal{A}_{\nabla}\left({ }^{1} \mathbb{R}_{2}\right)$ is the algebra of linear vector fields of the pseudoEuclidean plane ${ }^{1} \mathbb{R}_{2}$.

In turn, the geometric structure of the algebra $\mathcal{A}_{\nabla}\left(\mathbb{R}^{2}\right)$ of the linear vector fields with the structure of the matrix algebra $M_{2}(\mathbb{R})$ is determined by its generating vector fields, namely, by the radial and tangent vector fields

$$
\begin{aligned}
E=x \partial_{x}+y \partial_{y}, & I=y \partial_{x}-x \partial_{y} \\
J=y \partial_{x}+x \partial_{y}, & I J=x \partial_{x}-y \partial_{y}
\end{aligned}
$$

where the vector field $I J$ is orthogonal to $E$ in the metric of the pseudo-Euclidean plane $(x+y, x-y)$, and, hence, it is tangent to an equilateral hyperbola $x y=$ const. Thus, in this case $\mathcal{A}_{\nabla}\left(\mathbb{R}^{2}\right)$ is the algebra of linear vector fields of a system of planes $\left\{2^{1} \mathbb{R}_{2}, \mathbb{R}_{2}\right\} \cong \mathbb{R}^{2}$.

If referring to the Lie algebras $\mathcal{A}_{T}$, we should note that since a set of elements of this algebra is obtained by excluding the radial component of $E$ from the algebra $\mathcal{A}_{\nabla}$, then the geometry of the Lie algebra $\mathcal{A}_{T}$ is completely determined by the geometry of the corresponding tangent vector fields. In particular, if we consider the Lie algebra of the tangent vector fields of the algebra $\mathcal{A}_{\nabla}\left(\mathbb{R}_{4}\right)$ with the structure of the algebra of quaternions $\mathbb{H}$, then we would find that these vector fields are tangent to hyperspheres of the four-dimensional Euclidean space $\mathbb{R}_{4}$. Indeed, in this case

$$
\mathcal{A}_{T}\left(\mathbb{R}_{4}\right)=\left\langle\mathrm{i} \sigma_{1}, \mathrm{i} \sigma_{2}, \mathrm{i} \sigma_{3}\right\rangle_{\mathbb{R}}=\mathfrak{s u}(2)
$$

where $\sigma_{j}$ are the Pauli matrices, and $\mathrm{i} \sigma_{j}$ is the matrix representation of vector fields

$$
\begin{array}{ll}
\mathrm{i} \sigma_{1}: & x_{4} \partial x_{1}-x_{3} \partial x_{2}+x_{2} \partial x_{3}-x_{1} \partial x_{4} \\
\mathrm{i} \sigma_{2}: & x_{3} \partial x_{1}+x_{4} \partial x_{2}-x_{1} \partial x_{3}-x_{2} \partial x_{4} \\
\mathrm{i} \sigma_{3}: & x_{2} \partial x_{1}-x_{1} \partial x_{2}-x_{4} \partial x_{3}+x_{3} \partial x_{4}
\end{array}
$$

each of which is orthogonal to the radial vector field

$$
\sigma_{0}: \quad x_{1} \partial x_{1}+x_{2} \partial x_{2}+x_{3} \partial x_{3}+x_{4} \partial x_{4}
$$

in the metric of the Euclidean space $\mathbb{R}_{4}$. So, in this case $\mathcal{A}_{\nabla}\left(\mathbb{R}_{4}\right)$ is the algebra of linear vector fields in the Euclidean space $\mathbb{R}_{4}$. 
Similarly, if

$$
\mathcal{A}_{T}\left(\left\{3^{\{2\}} \mathbb{R}_{6}\right\}\right)=\left\langle\left[\mathrm{i} \sigma_{j}\right]_{k}\right\rangle_{\mathbb{R}}=s u(3)
$$

where $\left[\mathrm{i} \sigma_{j}\right]_{k}$ is the matrix representation of vector fields with zero components $\partial x_{2 k-1}$ and $\partial x_{2 k}$, namely

$$
\begin{array}{ll}
{\left[\mathrm{i} \sigma_{1}\right]_{1}:} & x_{6} \partial x_{3}-x_{5} \partial x_{4}+x_{4} \partial x_{5}-x_{5} \partial x_{6} \\
{\left[\mathrm{i} \sigma_{1}\right]_{2}:} & x_{6} \partial x_{1}-x_{5} \partial x_{2}+x_{2} \partial x_{5}-x_{1} \partial x_{6} \\
{\left[\mathrm{i} \sigma_{1}\right]_{3}:} & x_{4} \partial x_{1}-x_{3} \partial x_{2}+x_{2} \partial x_{3}-x_{1} \partial x_{4} \\
{\left[\mathrm{i} \sigma_{2}\right]_{1}:} & x_{5} \partial x_{3}+x_{6} \partial x_{4}-x_{3} \partial x_{5}-x_{4} \partial x_{6} \\
{\left[\mathrm{i} \sigma_{2}\right]_{2}:} & x_{5} \partial x_{1}+x_{6} \partial x_{2}-x_{1} \partial x_{5}-x_{2} \partial x_{6} \\
{\left[\mathrm{i} \sigma_{2}\right]_{3}:} & x_{3} \partial x_{1}+x_{4} \partial x_{2}-x_{1} \partial x_{3}-x_{2} \partial x_{4} \\
{\left[\mathrm{i} \sigma_{3}\right]_{1}:} & x_{4} \partial x_{3}-x_{3} \partial x_{4}-x_{6} \partial x_{5}+x_{5} \partial x_{6} \\
{\left[\mathrm{i} \sigma_{3}\right]_{2}:} & x_{2} \partial x_{1}-x_{1} \partial x_{2}-x_{6} \partial x_{5}+x_{5} \partial x_{6} \\
{\left[\mathrm{i} \sigma_{3}\right]_{3}:} & x_{2} \partial x_{1}-x_{1} \partial x_{2}-x_{4} \partial x_{3}+x_{3} \partial x_{4}
\end{array}
$$

where

$$
\left[\mathrm{i} \sigma_{3}\right]_{1}+\left[\mathrm{i} \sigma_{3}\right]_{3}=\left[\mathrm{i} \sigma_{3}\right]_{2}
$$

it is simple to verify that each of these vector fields is tangent to a hypersphere of the space ${ }^{\{2\}} \mathbb{R}_{6}$, i.e., of a six-dimensional semi-Euclidean space of defect two. Thus, in this case $\mathcal{A}_{\nabla}\left(\left\{3^{\{2\}} \mathbb{R}_{6}\right\}\right)$ is an algebra of linear vector fields of a system of semi-Euclidean spaces $\left\{3^{\{2\}} \mathbb{R}_{6}\right\}$.

In turn, if

$$
\mathcal{A}_{T}\left(\left\{2^{\{2\}} \mathbb{R}_{4}\right\}\right)=\left\langle\left(\sigma_{j}\right)_{3},\left(\mathrm{i} \sigma_{j}\right)_{3}\right\rangle_{\mathbb{R}}=\mathfrak{s l}_{2}(\mathbb{C})
$$

then the vector fields represented by the matrix $\left(\mathrm{i} \sigma_{j}\right)_{3}$ are tangent to hypersphere in the Euclidean space $\mathbb{R}_{4}$, and the vector fields represented by the matrix $\left(\sigma_{j}\right)_{3}$, are tangent to hyperspheres of the space ${ }^{2} \mathbb{R}_{4}$, i.e., the Euclidean spaces of index 2 which are, in other words, four-dimensional pseudo-Euclidean spaces with the metric signature $(2,2)$. In this case $\mathcal{A}_{\nabla}\left(\left\{2^{\{2\}} \mathbb{R}_{4}\right\}\right)$ is the algebra of linear vector fields of a system of the Euclidean spaces $\left\{{ }^{2} \mathbb{R}_{4}, \mathbb{R}_{4}\right\}$ or of an equivalent system of the semi-Euclidean space $\left\{2^{\{2\}} \mathbb{R}_{4}\right\}$.

Finally, let

$$
\mathcal{A}_{T}\left(\left\{4^{\{6\}^{6}} \mathbb{R}_{8}\right\}\right)=\left\langle\gamma_{0}, \mathrm{i} \gamma_{0}, \gamma_{[1,2,3]}, \mathrm{i} \gamma_{[1,2,3]}, \gamma_{0} \gamma_{[1,2,3]}, \mathrm{i} \gamma_{0} \gamma_{[1,2,3]}\right\rangle_{\mathbb{R}}=\mathfrak{s l}_{4}(\mathbb{C})
$$

where $\gamma_{[1,2,3]}$ is a set of seven Dirac matrices $\left(\gamma_{1}, \gamma_{2}, \gamma_{3}, \gamma_{1} \gamma_{2}, \gamma_{1} \gamma_{3}, \gamma_{2} \gamma_{3}, \gamma_{1} \gamma_{2} \gamma_{3}\right)$. Then one can notice that a half of this algebra, namely the vector fields represented by the matrices $\left(\gamma_{[1,2,3]}, \mathrm{i} \gamma_{0}, \mathrm{i} \gamma_{0} \gamma_{[1,2,3]}\right)$, is tangent to hyperspheres in the 
Euclidean space $\mathbb{R}_{8}$, while the other half of these vector fields, namely the vector fields represented by the matrices $\left(\mathrm{i} \gamma_{[1,2,3]}, \gamma_{0}, \gamma_{0} \gamma_{[1,2,3]}\right)$ are tangent to hyperspheres in the Euclidean space ${ }^{4} \mathbb{R}_{8}$. Thus, the geometric structure which would be interesting from the point of view of physical applications of the algebra of linear vector fields $\mathcal{A}_{\nabla}$ with the structure of the matrix algebra $M_{4}(\mathbb{C})$ is closely related to the geometry of the Euclidean spaces $\mathbb{R}_{8}$ and ${ }^{4} \mathbb{R}_{8}$. More precisely, in this case $\mathcal{A}_{\nabla}\left(\left\{4^{\{6\}} \mathbb{R}_{8}\right\}\right)$ is the algebra of linear vector fields of a system of the Euclidean spaces $\left\{3^{4} \mathbb{R}_{8}, \mathbb{R}_{8}\right\}$ or of an equivalent system of semi-Euclidean spaces $\left\{4^{\{6\}} \mathbb{R}_{8}\right\}$. Moreover, since

$$
\gamma_{5} \cdot \gamma_{\alpha}+\gamma_{\alpha} \cdot \gamma_{5}=0
$$

where $\alpha=0,1,2,3, \gamma_{5}=i \gamma_{0} \gamma_{1} \gamma_{2} \gamma_{3}$, then, in a four-dimensional vector space

$$
\left\langle\gamma_{5} \gamma_{0}, \gamma_{5} \gamma_{1}, \gamma_{5} \gamma_{2}, \gamma_{5} \gamma_{3}\right\rangle_{\mathbb{R}}=t^{*} \gamma_{5} \gamma_{0}+x^{*} \gamma_{5} \gamma_{1}+y^{*} \gamma_{5} \gamma_{2}+z^{*} \gamma_{5} \gamma_{3}
$$

a quadratic metric form

$$
s^{* 2}=-t^{* 2}+x^{* 2}+y^{* 2}+z^{* 2}
$$

is induced by the square

$$
\left(t^{*} \gamma_{5} \gamma_{0}+x^{*} \gamma_{5} \gamma_{1}+y^{*} \gamma_{5} \gamma_{2}+z^{*} \gamma_{5} \gamma_{3}\right)^{2}=\left(-t^{* 2}+x^{* 2}+y^{* 2}+z^{* 2}\right) E
$$

where $E$ is the identity matrix. At the same time, in a four-dimensional vector space

$$
\left\langle\gamma_{0}, \gamma_{1}, \gamma_{2}, \gamma_{3}\right\rangle_{\mathbb{R}}=t \gamma_{0}+x \gamma_{1}+y \gamma_{2}+z \gamma_{3}
$$

a quadratic metric form

$$
s^{2}=t^{2}-x^{2}-y^{2}-z^{2}
$$

is induced by the square

$$
\left(t \gamma_{0}+x \gamma_{1}+y \gamma_{2}+z \gamma_{3}\right)^{2}=\left(t^{2}-x^{2}-y^{2}-z^{2}\right) E
$$

The sum of these two squares induces a doublet (a Finsler product) of the Minkowski spaces with the metric form

$$
S^{2}=t^{2}-x^{2}-y^{2}-z^{2}-t^{* 2}+x^{* 2}+y^{* 2}+z^{* 2}
$$

Then, if we pass to the dual representation

$$
\begin{array}{ll}
\tau_{0}=\gamma_{0}+\gamma_{5} \gamma_{0}, & \tau_{1}=\gamma_{1}+\gamma_{5} \gamma_{1} \\
\tau_{2}=\gamma_{2}+\gamma_{5} \gamma_{2}, & \tau_{3}=\gamma_{3}+\gamma_{5} \gamma_{3} \\
\tau_{0}^{*}=\gamma_{0}-\gamma_{5} \gamma_{0}, & \tau_{1}^{*}=\gamma_{5} \gamma_{1}-\gamma_{1} \\
\tau_{2}^{*}=\gamma_{5} \gamma_{2}-\gamma_{2}, & \tau_{3}^{*}=\gamma_{5} \gamma_{3}-\gamma_{3}
\end{array}
$$


then the sum of four squares induces a metric form of the hyperbolic type, namely

$$
\begin{gathered}
\left(T \tau_{0}+T^{*} \tau_{0}^{*}\right)^{2}+\left(X \tau_{1}+X^{*} \tau_{1}^{*}\right)^{2}+\left(Y \tau_{2}+Y^{*} \tau_{2}^{*}\right)^{2}+\left(Z \tau_{3}+Z^{*} \tau_{3}^{*}\right)^{2}=T T^{*}+X X^{*} \\
+Y Y^{*}+Z Z^{*}=t^{2}-x^{2}-y^{2}-z^{2}-t^{* 2}+x^{* 2}+y^{* 2}+z^{* 2}
\end{gathered}
$$

As an example of a space which does not allow building an algebra of linear vector fields (due to the lack of tangent vector fields on the even-dimensional spheres), we can consider the Euclidean space $\mathbb{R}_{3}$. Here we should note that the system of the semi-Euclidean space $\left\{3^{\{1\}} \mathbb{R}_{3}\right\}$, which corresponds to the system of equations

$$
x_{1}^{2}+x_{2}^{2}=\text { const }, \quad x_{2}^{2}+x_{3}^{2}=\text { const }, \quad x_{1}^{2}+x_{3}^{2}=\text { const }
$$

already allows building an algebra of linear vector fields, its Lie algebra being isomorphic to the algebra $\mathfrak{s u}(2)$.

\section{The Dynamics of the Algebra of Vector Fields}

As a simple example of the dynamic representation of linear vector fields, we shall consider the local algebra of linear vector fields with the structure of the algebra of complex numbers. Since, due to the Cauchy-Riemann equations, the coordinate functions $x_{i}^{\prime}(x)$ imply the harmonic condition

$$
\frac{\partial^{2} x_{i}^{\prime}}{\partial x_{1}^{2}}+\frac{\partial^{2} x_{i}^{\prime}}{\partial x_{2}^{2}}=0
$$

we can regard a potential $u(x, y)$, which is defined on the plane $(x, y)$ and which satisfies the differential equation

$$
\frac{\partial^{2} u(x, y)}{\partial x^{2}}+\frac{\partial^{2} u(x, y)}{\partial y^{2}}=0
$$

as a dynamic representation of the local algebra of linear vector fields with the structure of the algebra of complex numbers. Similarly, in virtue of the CauchyRiemann equations, the local algebra of linear vector fields with the structure of the matrix algebra $M_{4}(\mathbb{C})$ has a dynamic representation in the form of a potential $u(z, \varphi)$ on an eight-dimensional cylindrical manifold with the coordinates $\left(z_{i}, \varphi_{i}\right)_{4}$, which satisfies the system of equations

$$
\frac{\partial^{2} u(z, \varphi)}{\partial z_{i}^{2}}+\frac{\partial^{2} u(z, \varphi)}{\partial \varphi_{i}^{2}}=0
$$


However, if we return to the original coordinates $\left(x_{j}\right)_{8}$, then, in these coordinates the system would have a similar form

$$
\Delta_{i}=\frac{\partial^{2} u(x)}{\partial x_{2 i-1}^{2}}+\frac{\partial^{2} u(x)}{\partial x_{2 i}^{2}}=0
$$

or an equivalent form

$$
\begin{aligned}
& \Delta_{1}+\Delta_{2}+\Delta_{3}+\Delta_{4}=0 \\
& \Delta_{1}-\Delta_{2}+\Delta_{3}-\Delta_{4}=0 \\
& \Delta_{1}+\Delta_{2}-\Delta_{3}-\Delta_{4}=0 \\
& \Delta_{1}-\Delta_{2}-\Delta_{3}+\Delta_{4}=0
\end{aligned}
$$

which implies that the potential $u(x)$ should satisfy the harmonic function condition in the Euclidean space and in pseudo-Euclidean spaces with the metric signature $(4,4)$. It should also be noted that we deal with the local (differential) conditions for the potential, and there are no global (integral) conditions which would be more interesting from the point of view of physics. However, let us try to guess which might be the vacuum solution of the Laplace equations.

First of all, note that since the function $f(x+\mathrm{i} y)=\ln \rho+\mathrm{i} \varphi$, where $\mathrm{i}$ is the generator of the algebra of complex numbers, $\rho=\sqrt{x^{2}+y^{2}}, \varphi=\arctan \frac{y}{x}$, is analytic in the sense of satisfying the Cauchy-Riemann conditions, and since the function $f(x+\mathrm{j} y)=\ln \rho+\mathrm{j} \varphi$, where $\mathrm{j}$ is the generator of the algebra of double numbers, $\rho=\sqrt{x^{2}-y^{2}}, \varphi=\ln \sqrt{\frac{x+y}{x-y}}$, is analytic in the sense of satisfying the hyperbolic Cauchy-Riemann conditions, then the functions of $\ln \rho$ and $\varphi$ are harmonic either in the Euclidean metric or in the metric of a pseudo-Euclidean plane. Furthermore, it should be noted that, if the Cartesian (linear) coordinates $\left(x^{\prime}, y^{\prime}\right)$ defined in the modified polar coordinates $\left(\ln \sqrt{x^{2}-y^{2}}, \ln \sqrt{\frac{x+y}{x-y}}\right)$ by using the identities $\left\{\left(x^{\prime}, 0\right),\left(0, y^{\prime}\right)\right\}=\{(\ln \rho, \varphi=0)\}$ are associated with the cylindrical manifold $\left(X=\ln \sqrt{x^{2}+y^{2}}, Y=\arctan \frac{y}{x}\right)$ by the relation

$$
x^{\prime}+y^{\prime}=X, \quad x^{\prime}-y^{\prime}=Y
$$

then, we can speak about two coordinate systems of the same cylindrical manifold $\mathbb{R} \times S^{1}$, homeomorphic to a punctured plane. 
Thus, since the functions

$$
\begin{aligned}
X & =\frac{1}{2} \ln \left(x_{1}^{2}+x_{2}^{2}\right), & \varphi_{X} & =X^{*}=\arctan \frac{x_{2}}{x_{1}} \\
Y & =\frac{1}{2} \ln \left(x_{3}^{2}+x_{4}^{2}\right), & \varphi_{Y} & =Y^{*}=\arctan \frac{x_{4}}{x_{3}} \\
Z & =\frac{1}{2} \ln \left(x_{5}^{2}+x_{6}^{2}\right), & \varphi_{Z} & =Z^{*}=\arctan \frac{x_{6}}{x_{5}} \\
T & =\frac{1}{2} \ln \left(x_{7}^{2}+x_{8}^{2}\right), & \varphi_{T} & =T^{*}=\arctan \frac{x_{8}}{x_{7}}
\end{aligned}
$$

satisfy the Cauchy-Riemann equations, each of these functions satisfies the system of Laplace equations, whereas the values of these functions are equal to the corresponding coordinates of the linear subspace $\left\langle\tau_{0}, \tau_{1}, \tau_{2}, \tau_{3}, \tau_{0}^{*}, \tau_{1}^{*}, \tau_{2}^{*}, \tau_{3}^{*}\right\rangle_{\mathbb{R}}$ of the algebra $M_{4}(\mathbb{C})$ which induces a quadratic metric form

$$
S^{2}=t^{2}-x^{2}-y^{2}-z^{2}-t^{* 2}+x^{* 2}+y^{* 2}+z^{* 2}
$$

of the space ${ }^{4} \mathbb{R}_{8}$ that contains a pair of Minkowski spaces, namely, ${ }^{3} \mathbb{R}_{4}$ and ${ }^{1} \mathbb{R}_{4}$. Thus, by using the following substitution of variables

$$
\begin{array}{llrl}
T & =t+t^{*}, & & T^{*}=t-t^{*} \\
X & =x^{*}+x, & & X^{*}=x^{*}-x \\
Y & =y^{*}+y, & & Y^{*}=y^{*}-y \\
Z & =z^{*}+z, & Z^{*} & =z^{*}-z
\end{array}
$$

we can reduce this quadratic to the hyperbolic form

$$
S^{2}=T T^{*}+X X^{*}+Y Y^{*}+Z Z^{*}
$$

where the coordinates of the $\left(X, X^{*}, Y, Y^{*}, Z, Z^{*}, T, T^{*}\right)$ are isotropic coordinates of the space ${ }^{4} \mathbb{R}_{8}$, and the scalar product associated with this hyperbolic form, is as follows

$$
\begin{aligned}
2 a b=a_{T} b_{T^{*}}+a_{T^{*}} b_{T}+a_{X} b_{X^{*}} & +a_{X^{*}} b_{X} \\
& +a_{Y} b_{Y^{*}}+a_{Y^{*}} b_{Y}+a_{Z} b_{Z^{*}}+a_{Z^{*}} b_{Z} .
\end{aligned}
$$

A pair of isotropic coordinates of the pseudo-Euclidean plane is the simplest system of coordinates of a corresponding cylinder $\mathbb{R} \times S^{1}$ or of a torus $S^{1} \times S^{1}$, which are obtained by compactifying the plane as a result of the compactification of, respectively, one or two isotropic coordinates. However, the full compactification (the compactification of two isotropic coordinates) of all pseudo-Euclidean planes 
of the space ${ }^{1} \mathbb{R}_{4}$ turns this space into a toroidal manifold $S^{3} \times S^{1}$. Indeed, since the space of ${ }^{1} \mathbb{R}_{4}$ is given by the set of pseudo-Euclidean planes, such as ${ }^{1} \mathbb{R}_{4}$ (where the prime index of the coordinate $x^{\prime *}$ is the index of a set of centre-symmetric lines of the Euclidean space $\left(x^{*}, y^{*}, z^{*}\right)$, i.e., this index runs over the projective plane $\mathbb{R P}^{2}$ ), then we obtain a topological equivalence ${ }^{1} \mathbb{R}_{4} \cong \mathbb{R P}^{2} \times{ }^{1} \mathbb{R}_{2}$, whereas the compactified space ${ }^{1} \mathbb{R}_{4}$ is homeomorphic to $\mathbb{R P}^{2} \times S^{1} \times S^{1} \cong S^{3} \times S^{1}$. Note here that, as a result of the compactification of the space ${ }^{1} \mathbb{R}_{4}$, this space turns into a cone isotropic two adjoining spheres $S^{3}$. Similarly, one can compactify the Minkowski space $(t, x, y, z)$. In turn, as ${ }^{4} \mathbb{R}_{8} \cong \mathbb{R P}^{3} \times \mathbb{R P}^{3} \times{ }^{1} \mathbb{R}_{2}$, the result of the compactifications of two Minkowski spaces will be the product of spheres $\mathbb{R} P^{3} \times \mathbb{R P}^{3} \times S^{1} \times S^{1} \cong S^{4} \times S^{4}$, while the isotropic cone of the Euclidean space ${ }^{4} \mathbb{R}_{8}$ will be compactified to two contiguous spaces $\mathbb{R P}^{3} \times \mathbb{R P}^{3} \times S^{1}$.

From the point of view of mathematical modelling of physical phenomena, space ${ }^{4} \mathbb{R}_{8}$ is of particular interest. Indeed, suppose that in this space we have a dynamic flow of a continuous medium with the vector field $\mathbf{v}$ of the test particle velocities being divergence-free everywhere. However, this vector field $\mathbf{v}$ may have some topological singularities were the flow lines of the vector field compactify on the corresponding toroidal manifold (in the simplest case, to a circle), which happens when $\mathbf{v}^{2}=0$. In addition, let the path integral

$$
S=\int_{L} \mathbf{v}^{*} \mathrm{~d} l
$$

of the scalar product between the co-vectorial velocity field $\mathbf{v}^{*}$ of test particles and the differential path element $\mathrm{d} l$ along the path of a topological singularity moving across the vector field in ${ }^{4} \mathbb{R}_{8}$ (not taking into account the vector fields of the topological singularity itself) be a measure of the amount of the proper rotation of this topological singularity, the rotation being caused by the action of the flow. Then this curvilinear integral can be associated with the action along a world line of a material point moving in the Minkowski space ${ }^{3} \mathbb{R}_{4}$, this world line being obtained by orthogonal projection of the trajectory from ${ }^{4} \mathbb{R}_{8}$ to ${ }^{3} \mathbb{R}_{4}$. On the other hand, if the Minkowski subspace ${ }^{3} \mathbb{R}_{4}$ of ${ }^{4} \mathbb{R}_{8}$ is not defined globally (by a single basis), but it is defined only locally (by the reference bundle of a four-dimensional manifold), then we can define the covariant derivative of a vector field tangent to the path specified in ${ }^{4} \mathbb{R}_{8}$ along the world lines of the corresponding global manifold as the orthogonal projection of the derivative of this field on a given manifold.

In addition, let the covector velocity field of the particle flow be a potential, i.e., $\mathbf{v}^{*}=\nabla u$. Then, demanding the implementation of the following variational equation

$$
\delta \int \nabla^{2} u \mathrm{~d}^{8} x=0
$$


that provides the uniformity of the flow, we obtain a necessary condition in the form of the differential equation

$$
\frac{\partial^{2} u}{\partial t^{2}}-\frac{\partial^{2} u}{\partial x^{2}}-\frac{\partial^{2} u}{\partial y^{2}}-\frac{\partial^{2} u}{\partial z^{2}}=\frac{\partial^{2} u}{\partial t^{* 2}}-\frac{\partial^{2} u}{\partial x^{* 2}}-\frac{\partial^{2} u}{\partial y^{* 2}}-\frac{\partial^{2} u}{\partial z^{* 2}} .
$$

We should keep in mind that each pair of coordinates $\left(t, t^{*}\right),\left(x, x^{*}\right),\left(y, y^{*}\right),\left(z, z^{*}\right)$ corresponds to a coordinate system of a cylindrical or toroidal manifold and, therefore, equation (91) should be accompanied by the condition of periodicity of the gradient of the potential $\nabla u\left(T, X, Y, Z, T^{*}, X^{*}, Y^{*}, Z^{*}\right)$ along the isotropic coordinates with the asterisks or along all the isotropic coordinates. We note here that, in isotropic coordinates, equation (91) takes the form

$$
\frac{\nabla^{2} u}{\partial T \partial T^{*}}+\frac{\nabla^{2} u}{\partial X \partial X^{*}}+\frac{\nabla^{2} u}{\partial Y \partial Y^{*}}+\frac{\nabla^{2} u}{\partial Z \partial Z^{*}}=0 .
$$

In turn, a stronger requirement of stability (equilibrium) of the flow, which is equivalent to the local extremality of the curvilinear integral along an arbitrary path

$$
\delta S=\delta \int_{L} \nabla u \mathrm{~d} l=0
$$

yields us the necessary condition, which consists of the fact that the level surfaces of the potential function $u(x)$ is minimal in the space ${ }^{4} \mathbb{R}_{8}$. That is, the mean curvature of these surfaces must be vanishing. In other words, the variational equation (93) has a solution given by the differential equation

$$
\mathrm{d} \star n(x)=0
$$

where $n(x)=\frac{\nabla u(x)}{|\nabla u(x)|}$, and the star denotes the Hodge operator. However, if we are interested in the dynamical characteristics of the vector field, we should refer to the same variational equation (93), provided the condition that what is varied here is the trajectory of a topological singularity moving in ${ }^{4} \mathbb{R}_{8}$, whereas the gradient of the potential plays the role of an external field.

Let us now consider the classical limit of a particular trajectory $L$, such that the coordinates of the trajectory satisfy the system of equations

$$
t-t^{*}=0, \quad x^{*}-x=0, \quad y^{*}-y=0, \quad z^{*}-z=0
$$

which implies that $L$ reduce to a path $S$ in the space ${ }^{3} \mathbb{R}_{4}$ and the mirror path $S^{*}$ in the space ${ }^{1} \mathbb{R}_{4}$. In this case, the equation (93) is reduced to the equation in the classical limit

$$
\delta\left[m \int_{S} \operatorname{pr}_{\mathbb{R}_{4}} \nabla u \mathrm{~d} s+q \int_{S^{*}} \operatorname{pr}_{\mathbb{R}_{4}} \nabla u \mathrm{~d} s^{*}\right]=0
$$


where $m, q$ are the characteristics of the topological singularity (particle), and the fields $\operatorname{pr}_{3 \mathbb{R}_{4}} \nabla u, \operatorname{pr}_{1 \mathbb{R}_{4}} \nabla u$ - are the orthogonal projections of the gradient of the potential in the corresponding Minkowski space. We now construct a pseudoRiemannian manifold $\left(t^{\prime}, x^{\prime}, y^{\prime}, z^{\prime}\right)$, such that $t^{\prime}=t^{* *}, x^{\prime}=x^{* *}, y^{\prime}=y^{\prime *}, z^{\prime}=z^{\prime *}$, the metric is $g^{i j}(\mathbf{x})=\left(\nabla x_{i}(\mathbf{x}), \nabla x_{j}^{\prime}(\mathbf{x})\right)$ (where $\mathbf{x} \in{ }^{4} \mathbb{R}_{8}, x_{1}, \ldots, x_{4}=t, x, y, z$ ) which is induced by an inner product of the space ${ }^{4} \mathbb{R}_{8}$; and the length of the coordinate increments in the tangent (locally pseudo-Euclidean) space $\left(\Delta t^{\prime}, \Delta x^{\prime}\right.$, $\left.\Delta y^{\prime}, \Delta z^{\prime}\right)$ of the pseudo-Riemannian manifold is calculated by the formula

$$
\begin{aligned}
\left|\Delta x_{i}^{\prime}\right|= & \nabla x_{i}^{\prime}(\mathbf{x}) \cdot \Delta \mathbf{x} \\
= & \frac{\partial x_{i}^{\prime}(\mathbf{x})}{\partial x_{1}} \Delta x_{1}-\frac{\partial x_{i}^{\prime}(\mathbf{x})}{\partial x_{2}} \Delta x_{2}-\frac{\partial x_{i}^{\prime}(\mathbf{x})}{\partial x_{3}} \Delta x_{3}-\frac{\partial x_{i}^{\prime}(\mathbf{x})}{\partial x_{4}} \Delta x_{4} \\
& -\frac{\partial x_{i}^{\prime}(\mathbf{x})}{\partial x_{1}^{*}} \Delta x_{1}^{*}+\frac{\partial x_{i}^{\prime}(\mathbf{x})}{\partial x_{2}^{*}} \Delta x_{2}^{*}+\frac{\partial x_{i}^{\prime}(\mathbf{x})}{\partial x_{3}^{*}} \Delta x_{3}^{*}+\frac{\partial x_{i}^{\prime}(\mathbf{x})}{\partial x_{4}^{*}} \Delta x_{4}^{*} \\
= & \left(\frac{\partial x_{i}^{\prime}(\mathbf{x})}{\partial x_{1}}-\frac{\partial x_{i}^{\prime}(\mathbf{x})}{\partial x_{1}^{*}}\right) \Delta x_{1}-\left(\frac{\partial x_{i}^{\prime}(\mathbf{x})}{\partial x_{2}}-\frac{\partial x_{i}^{\prime}(\mathbf{x})}{\partial x_{2}^{*}}\right) \Delta x_{2} \\
& -\left(\frac{\partial x_{i}^{\prime}(\mathbf{x})}{\partial x_{3}}-\frac{\partial x_{i}^{\prime}(\mathbf{x})}{\partial x_{3}^{*}}\right) \Delta x_{3}-\left(\frac{\partial x_{i}^{\prime}(\mathbf{x})}{\partial x_{4}}-\frac{\partial x_{i}^{\prime}(\mathbf{x})}{\partial x_{4}^{*}}\right) \Delta x_{4}
\end{aligned}
$$

However, since it is assumed that $t^{\prime}=u$, the scalar product of $\nabla u \mathrm{~d} l$ taken from the integrand of the equation (93) is equal to $\left|\Delta t^{\prime}\right|$ and, therefore, the integral in equation (93) is equal to the length of the world line of the topological singularity in a pseudo-Riemannian four-manifold $\left(t^{\prime}, x^{\prime}, y^{\prime}, z^{\prime}\right)$, i.e., it is equal to proper time of this topological singularity.

Thus, the potential function

$$
u_{0}\left(x_{1}, \ldots, x_{8}\right)=t_{\varphi}=\frac{\mathrm{e}^{\varphi}}{2}(T+X+Y+Z)+\frac{\mathrm{e}^{-\varphi}}{2}\left(T^{*}+X^{*}+Y^{*}+Z^{*}\right)
$$

is proposed to be used as a vacuum potential without taking into account the local (field) changes of the shape of this potential, whereas the parameter $\varphi$ is proposed to be regarded as responsible for the global (evolutionary) changes of the vacuum potential shape. Note also that formally the vacuum potential can be represented by a complex analytic function

$$
\bar{u}_{0}\left(x_{1}, \ldots, x_{8}\right)=\frac{\mathrm{e}^{\varphi}}{2}(T+X+Y+Z)+\mathrm{i} \frac{\mathrm{e}^{-\varphi}}{2}\left(T^{*}+X^{*}+Y^{*}+Z^{*}\right) .
$$

In this case, we simply assume that

$$
u_{0}\left(x_{1}, \ldots, x_{8}\right)=\operatorname{Re} \bar{u}_{0}\left(x_{1}, \ldots, x_{8}\right)+\operatorname{Im} \bar{u}_{0}\left(x_{1}, \ldots, x_{8}\right) .
$$


However, if, in addition to linear harmonic functions, we are to consider the harmonic functions generated by a linear combination of the real and imaginary components of the rational complex linear functions, these can be regarded as particlelike solutions of a system of Laplace equations. For example, the function

$$
\bar{u}\left(x_{1}, \ldots, x_{8}\right)=\bar{u}_{0}+\frac{1}{\rho+\mathrm{i} \rho^{*}}
$$

wherein $\rho=\sqrt{x^{2}+y^{2}+z^{2}}, \rho^{*}=\sqrt{x^{* 2}+y^{* 2}+z^{* 2}}$, with the proviso that

$$
\begin{array}{ll}
x=T-X+Y-Z, & x^{*}=T^{*}-X^{*}+Y^{*}-Z^{*} \\
y=T+X-Y-Z, & y^{*}=T^{*}+X^{*}-Y^{*}-Z^{*} \\
z=T-X-Y+Z, & z^{*}=T^{*}-X^{*}-Y^{*}+Z^{*}
\end{array}
$$

can be considered a complex extension of the Newtonian potential of a material point.

On the other hand, let the radius of the sphere be

$$
\rho=\sqrt{x_{1}^{2}+x_{2}^{2}+x_{3}^{2}+x_{4}^{2}+x_{5}^{2}+x_{6}^{2}+x_{7}^{2}+x_{8}^{2}}
$$

the radius of a pseudo-sphere being

$$
R=\sqrt{x_{1}^{2}+x_{2}^{2}+x_{3}^{2}+x_{4}^{2}-x_{5}^{2}-x_{6}^{2}-x_{7}^{2}-x_{8}^{2}}
$$

and hyperbolic angle being $\varphi=\ln \frac{\rho}{R}$. These define a complex potential function

$$
\bar{u}\left(x_{1}, \ldots, x_{8}\right)=\ln R+\mathrm{i} \varphi .
$$

Then, we can see this as an evolving vacuum potential of the Universe, where the evolution parameter $\tau$ coincides with the hyperbolic angle $\varphi$. And, since it is implicitly assumed that radius $\rho$ of the sphere is a constant which is independent on the evolution parameter $\tau$, then

$$
\tau=\ln \frac{1}{R}
$$

We note here that the level surface of the potential function $u(x)=\ln R$ is homeomorphic to the product $S^{3} \times \mathbb{R}^{4}$.

In conclusion, it should be noted that all these analogies with the physical world require further study, including also the possibility of expanding the space of partial solutions of our system of the Laplace equations. It is also necessary to pay attention to the following transformation of a complex vector

$$
\psi=\left(\begin{array}{c}
\psi_{1} \\
\psi_{2} \\
\psi_{3} \\
\psi_{4}
\end{array}\right)=\left(\begin{array}{c}
\xi_{x}+\mathrm{i} \xi_{t^{*}} \\
\xi_{y}+\mathrm{i} \xi_{z} \\
\xi_{x^{*}}+\mathrm{i} \xi_{t} \\
\xi_{y^{*}}+\mathrm{i} \xi_{z^{*}}
\end{array}\right)
$$


where

$$
\left(\psi^{*} \gamma_{0}, \psi\right)=\xi^{2}=\xi_{t}^{2}-\xi_{x}^{2}-\xi_{y}^{2}-\xi_{z}^{2}-\xi_{t^{*}}^{2}+\xi_{x^{*}}^{2}+\xi_{y^{*}}^{2}+\xi_{z^{*}}^{2}=1,-1,0
$$

which induces an isometric transformations of a real linear space ${ }^{4} \mathbb{R}_{8}$. As for the Lorentz transformations of the Minkowski space $(t, x, y, z)$, we have to note that these transformations have a bispinor representation realized in the form of the algebra of vector fields tangent to the space ${ }^{4} \mathbb{R}_{8}$ (which is equivalent to the algebra of tangent vector fields of $\left\{{ }^{2} \mathbb{R}_{4}, \mathbb{R}_{4}\right\}$ isomorphic to $\mathfrak{s l}_{2}(\mathbb{C})$ ). The rotation of the Euclidean space $(x, y, z)$ is reduced to a spinor representation implemented in the algebra of vector fields tangent to the space $\mathbb{R}_{4}$, the latter being isomorphic to $\mathfrak{s u}(2)$. Thereby, the bispinor $\psi$ has the meaning of complexified directional vector of the congruence of straight parallel trajectories of topological singularities and, in combination with the rotation angle (phase action) of the topological singularities, this makes it possible to construct a bispinor wave function of the congruence of the topological singularity trajectories $\Psi=\psi \mathrm{e}^{\mathrm{i} S}$, where $S$ is the action of the topological singularities depending on its trajectory. Moreover, if we regard the vector $\xi$ as a vector-valued functions $\xi(t, x, y, z)$ with its domain in the Minkowski space, which delivers an extremum of the functional

$$
\int \xi(x) \cdot \nabla \xi(x) \mathrm{d}^{4} x
$$

and, hence, it is a solution of the variational equation

$$
\delta \int \xi \cdot\left(\partial_{t} \xi^{t}+\partial_{x} \xi^{x}+\partial_{y} \xi^{y}+\partial_{z} \xi^{z}\right) \mathrm{d}^{4} x=0
$$

where $\xi^{t}, \xi^{x}, \xi^{y}, \xi^{z}$ are the values of the corresponding tangent vector fields of the space ${ }^{4} \mathbb{R}_{8}$ at the point $\xi$, namely $\xi^{t}=\gamma_{0} \xi$, $\xi^{x}=\gamma_{1} \xi$, $\xi^{y}=\gamma_{2} \xi$, $\xi^{z}=\gamma_{3} \xi$, with $\gamma_{0}, \gamma_{1}, \gamma_{2}, \gamma_{3}$ being the real Dirac gamma matrices, then a complex vector function $\psi(t, x, y, z)$ satisfies the Dirac equation.

On the other hand, since the topological singularities are compact geometrical objects (circles, eight-shaped twisted circles, tori, etc.) lying on the compactified isotropic cone of the Euclidean space ${ }^{4} \mathbb{R}_{8}$, then it makes sense to explore the symmetries of these compact geometrical objects from the point of view of their correspondence to the global gauge symmetries of the Standard Model of particle physics. We note here that an arbitrary circle is associated with an arbitrary complex number of unit length, a two-dimensional torus (the product of two circles) can be associated with a pair of unit complex numbers, a three-dimensional torus can be associated with a triplet of unit complex numbers, and so on. The proper rotation of a unit circle $z=\mathrm{e}^{\mathrm{i} \varphi}$ can be presented as an action of the group $U(1)$. 
However, if the rotation of a pair of unit circles $\left(z_{1}=\mathrm{e}^{\mathrm{i} \varphi_{1}}, z_{2}=\mathrm{e}^{\mathrm{i} \varphi_{2}}\right)$ is representing the action of the orthogonal group $\mathrm{SO}_{\mathbb{R}}(2)$, then an arbitrary composition of rotations of these circle pairs, together with their proper rotations, represented by the action of unimodular diagonal matrices, all the matrix elements of which have modulus one, i.e., $\operatorname{diag}\left[\mathrm{e}^{\mathrm{i} \alpha_{1}}, \mathrm{e}^{\mathrm{i} \alpha_{2}}\right]$, given the condition $\alpha_{1}+\alpha_{2}=2 \pi k$, belongs to the unitary transformation group $\mathrm{SU}(2)$. At the same time, an arbitrary composition of the rotations of three unit circles $\left(z_{1}=\mathrm{e}^{\mathrm{i} \varphi_{1}}, z_{2}=\mathrm{e}^{\mathrm{i} \varphi_{2}}, z_{3}=\mathrm{e}^{\mathrm{i} \varphi_{3}}\right)$, represented by the action of $\mathrm{SO}_{\mathbb{R}}(3)$, and their proper rotations represented by the action of diag $\left[\mathrm{e}^{\mathrm{i} \alpha_{1}}, \mathrm{e}^{\mathrm{i} \alpha_{2}}, \mathrm{e}^{\mathrm{i} \alpha_{3}}\right]$, where $\alpha_{1}+\alpha_{2}+\alpha_{3}=2 \pi k$, belongs to the group of unitary transformations $\mathrm{SU}(3)$. Thus, given the identity

$$
n^{2}-1=\frac{(n-1) n}{2}+(n-1)+\frac{(n-1) n}{2}
$$

meaning the equality of the dimensions of the groups $\mathrm{SU}(n)$ and of the group formed of the products of the group $\mathrm{SU}(n)$, where the first and the third elements belong to the group $\mathrm{SO}_{\mathbb{R}}(n)$, and the second element belongs to the group $\operatorname{diag}\left[\mathrm{e}^{\mathrm{i} \alpha_{1}}, \ldots, \mathrm{e}^{\mathrm{i} \alpha_{n}}\right]$, given the condition $\alpha_{1}+\cdots+\alpha_{n}=2 \pi k$, one can conclude that all these rotation of a circle, of a two-torus and of a three-torus form a group $U(1) \times \mathrm{SU}(2) \times \mathrm{SU}(3)$, which coincides with the group of unbroken symmetries model, which describes both the strong, weak and electromagnetic interactions. Thus, the group of the unbroken symmetries describes the symmetries of the circle, two-torus and three-torus lying on the compactified isotropic cone of the space ${ }^{1} \mathbb{R}_{4}$, that is, on the two touching spheres $S^{3}$.

In general, it should be understood that the isotropic coordinates $(T, X, Y, Z)$ of the Minkowski space $(t, x, y, z)$, as well as the isotropic coordinates $\left(T^{*}, X^{*}\right.$, $\left.Y^{*}, Z^{*}\right)$ of the space $\left(t^{*}, x^{*}, y^{*}, z^{*}\right)$, where

$$
\begin{array}{rlrl}
t & =T+X+Y+Z, & & x^{*}=T^{*}+X^{*}+Y^{*}+Z^{*} \\
x=T-X+Y-Z, & & x^{*}=T^{*}-X^{*}+Y^{*}-Z^{*} \\
y=T+X-Y-Z, & y^{*}=T^{*}+X^{*}-Y^{*}-Z^{*} \\
z=T-X-Y+Z, & z^{*}=T^{*}-X^{*}-Y^{*}+Z^{*}
\end{array}
$$

are compactified. But this compactification happen as a result of the evolution of the vacuum potential (due to the presence in it of the multipliers $\mathrm{e}^{\varphi}$ and $\mathrm{e}^{-\varphi}$ ), the diameter of one of the compactified isotropic cones being increasing, whereas the diameter of the other decreasing. At the same time, the vacuum potential is transformed not only globally, resulting in the formation and evolution of its globally minimal level surfaces, but also locally, either as a result of the formation and motion of its locally minimal level surfaces without topological singularities (fields), or as a result of the formation and motion of its local minimal level surfaces with 
singularities / topological singularities (particles), or as a result of random fluctuations in the dynamic flow of a continuous medium.

\section{References}

[1] Tyshkevich R. and Fedenko A., Linear Algebra and Analytic Geometry (in Russian), Vyisheyshaya Shkola, Minsk 1976.

[2] Gelfand I., Lectures on Linear Algebra, Dover, New York 1998.

[3] Vinberg E., A Course in Algebra, Graduate Studies in Mathematics vol. 56, AMS, Providence 2003.

[4] Gorbuzov V., Mathematical Analysis: Field Theory (in Russian), Grodno State University, Grodno 2000.

[5] Efimov N. and Rozendorn E., Linear Algebra and Multidimensional Geometry, Mir, Moscow 1975.

[6] Rosenfeld B., Non-Euclidean Geometries, Gostekhizdat, Moscow 1955.

[7] Novikov S. and Taimanov I., Modern Geometric Structures and Fields, Graduate Studies in Mathematics vol. 71, AMS, Providence 2006.

[8] Hestenes D., Space-Time Algebra, Gordon \& Breach, New York 1966.

[9] Kassandrov V., Algebraic Structure of Space-Time and Algebrodynamics (in Russian), People Fried. Univ. Press, Moscow 1992.

[10] Rumer Y. and Fet A., The Theory of Unitary Symmetry (in Russian), Nauka, Moscow 1970.

[11] Pavlov D., Generalization of Scalar Product Axioms, Hypercomplex Numbers in Geometry and Physics 1 (2004) 5-18.

[12] Siparov S., On the Anisotropic Geometrodynamics (in Russian), Hypercomplex Numbers in Geometry and Physics 10 (2008) 64-75.

Igor V. Bayak

Khimvolokno Technology Complex, JSC Grodno Azot

100 Kosmonavtov, Grodno 230013, BELARUS

E-mail address: bayak@tut.by 